\title{
INDICADORES DA MANUTENÇÃO: ESTUDO DA SUA EFICIÊNCIA NO SETOR DE ENGENHARIA CLÍNICA ${ }^{1}$
}

\author{
MAINTENANCE INDICATORS: STUDY OF ITS \\ EFFICIENCY IN THE CLINICAL ENGINEERING SECTOR
}

\section{Marcella Wayss Darold², Liliane Alves Pereira ${ }^{3}$ e Luiz Fernando Rodrigues Junior ${ }^{4}$}

\section{RESUMO}

Com o passar dos anos, as tecnologias na área da saúde, que estão em constante evolução, foram incorporadas aos estabelecimentos assistenciais de saúde (EAS) de forma rápida, fazendo os EAS implementarem novas técnicas para gerir com mais eficiência o funcionamento dos dispositivos, o que traz segurança e garantia na operação. O setor de engenharia clínica de um hospital desempenha suas atividades baseando-se em objetivos, metas e resultados e, com isso, os indicadores são uma ferramenta de medição que contribuem para a avaliação da qualidade dos serviços e apresentam dados que mostram o progresso do setor. Dessa forma, realizou-se uma revisão bibliográfica das aplicações dos indicadores e alguns indicadores que são utilizados no setor de engenharia clínica. As buscas foram feitas em bases de dados eletrônicas com alguns critérios de inclusão. Após a escolha dos artigos, todos foram revisados e selecionados de acordo com o critério de inclusão. Foi feita uma listagem com os indicadores encontrados e, para montar a lista, os indicadores foram divididos em classes. Em seguida, os indicadores foram colocados nas classes de tempo, custo e qualidade. Foram selecionados quatorze indicadores de tempo, treze indicadores de custo e quinze indicadores de qualidade, e a aplicação de alguns foi discutida ao longo dos resultados, apresentando a sua eficiência. Os indicadores colaboram muito para o desempenho do estabelecimento e precisam ser adaptáveis a cada realidade. A gestão deve sempre fazer uma análise crítica dos seus resultados ao longo do tempo, pois os indicadores são elaborados de acordo com a necessidade e o objetivo do setor.

Palavras-chave: hospital, tecnologias em saúde, gestão.

\section{ABSTRACT}

Over the years, technologies in the health area are constantly evolving and being quickly incorporated into health care facilities (EAS), making the EAS implement new techniques to better manage the operation of devices, bringing safety and security to the operation. The clinical engineering sector of a hospital carries out its activities based on objectives, goals and results, and with this the indicators are a measurement tool that contribute to the assessment of the quality of services and present data that show the progress of the sector. Thus, a literature review of the applications of indicators and some indicators that are used in the clinical engineering sector was carried out. Searches were performed in an electronic database with some inclusion criteria, after selecting the articles, all articles were reviewed and selected according to the inclusion criteria, a list was made with the indicators found in them, to assemble the list the indicators were divided in classes. Then the indicators were placed in time, cost and quality classes, fourteen time indicators, thirteen cost indicators and fifteen quality indicators were selected, the application of some was discussed along the results

\footnotetext{
1 Projeto de extensão.

2 Discente do Curso de Engenharia Biomédica-marcella.darold@ufn.edu.br

3 Co-orientadora do Curso de Enfermangem-liliane.pereira@ufn.edu.br

4 Orientador do Curso de Engenharia Biomédica-luiz.fernando@ufn.edu.br
} 
showing their efficiency. The indicators contribute a lot to the establishment's performance, they need to be adaptable to the reality of the establishment. Management must always make a critical analysis of its results over time, as the indicators are prepared according to the needs and objectives of the sector.

Keywords: hospital, health technologies, management.

\section{INTRODUÇÃO}

No decorrer dos anos, a incorporação de tecnologias na área da saúde vem ocorrendo de forma acelerada no Brasil e no mundo, e a inserção dessas tecnologias nos estabelecimentos assistências de saúde (EAS) tem um grande impacto financeiro. A aquisição de novas tecnologias envolve diversas áreas dos EAS, pois precisa de uma gestão eficiente e que tenha a perspectiva de vários cenários, pois, em um cenário, tem-se o paciente e o operador do equipamento e, no outro os gestores do estabelecimento, que têm em vista os custos e recursos financeiros (NOVAES; DE SOÁREZ, 2020).

Com o avanço dessas tecnologias, a gestão dos EAS precisa implementar novas técnicas para ter um bom funcionamento, além de segurança e garantia na operação do equipamento, conforme descrito na RDC 509/2021 (AGÊNCIA NACIONAL DE VIGILÂNCIA SANITÁRIA, 2021). O gerenciamento dos equipamentos médicos engloba diversos processos, que vão desde a aquisição, avaliação, manutenção até o descarte desses dispositivos (ARAÚJO, 2020; ASSOCIAÇÃO BRASILEIRA DE NORMAS TÉCNICAS, 2011).

As tecnologias em saúde estão sempre em constante evolução e desenvolvimento, de forma a propiciar maior segurança e qualidade para as práticas de atendimento dos profissionais, porém essa constante melhoria acarreta maior complexidade do uso e entendimento destas. Com isso, a gestão hospitalar precisa manter um quadro de aprimoramentos e treinamentos para que os profissionais e os técnicos fiquem cada vez mais capacitados (MAGGI et al., 2019; ORTIZ; ARAÚJO, 2020). Como descrito na RDC 509/2021, os equipamentos de saúde devem funcionar de forma segura e confiável. Para isso, eles precisam de uma gestão de manutenção eficaz (AGÊNCIA NACIONAL DE VIGILÂNCIA SANITÁRIA, 2021). Com a evolução das tecnologias, a manutenção também deve evoluir, tanto em nível técnico como de gestão, garantindo maior qualidade de uso ao longo do ciclo de vida desses dispositivos, com vista à segurança e eficácia no seu uso, pois qualquer falha pode acarretar danos aos pacientes e aos profissionais de saúde (IADANZA et al., 2019; SEZDI, 2016; SHAMAYLEH; AWAD; FARHAT, 2020).

A manutenção está totalmente relacionada ao setor de engenharia clínica de um hospital, pois esse setor tem um aspecto fundamental nas atividades que envolvem recursos humanos e fi- 
nanceiros importantes. Por isso, a avaliação da eficiência da manutenção está diretamente ligada ao aprimoramento do uso dos recursos e a gestão da engenharia clínica do hospital (GONNELLI et al., 2017; IADANZA et al., 2019; WANG et al., 2010).

Diversos setores na indústria trabalham baseando-se em objetivos, metas e resultados, assim como o setor de engenharia clínica de um hospital. Nesse contexto, uma maneira eficaz de demonstrar que o resultado foi alcançado como o planejado são os indicadores. Com a aplicação deles, o setor obtém dados determináveis que mostram o progresso em todas as perspectivas (SILVA; LUCENA; TOSCANO, 2015). Para Cardoso e Calil (2000), indicadores são medições de várias dimensões que contribuem na avaliação da qualidade dos serviços que o setor ou terceiros realizam, eles são fundamentais para analisar a qualidade, manutenção dos equipamentos, planejamento e redução de custos e o rendimento operacional. Essas medições surgiram com intuito de ajudar em diagnósticos para ver o que está dando certo, o que precisa melhorar e propor novas medidas (ARAÚJO, 2020).

Os indicadores são aplicados dentro de processos de gestão para mensurar algum produto ou processo e servem como controle das práticas realizadas. De posse dessa definição, é claro o entendimento da necessidade de indicadores para a manutenção, pois, como descrito previamente, a manutenção visa manter a qualidade e segurança dos equipamentos. Quando fala-se de indicadores para processos de gestão, é comum encontrar o termo em inglês KPI, que significa Key Performance Indicators ou, em tradução livre, Indicadores Chave de Performance (OMAR; IBRAHIM; OMAR, 2017).

Existem diversos indicadores que podem ser aplicados nos processos de manutenção do ambiente hospitalar, sendo importante, primeiramente, entender o que se espera do setor responsável por esse serviço. Além disso, deve-se procurar definir indicadores factíveis, objetivos e que demonstrem o que é esperado para medir os serviços de manutenção (OMAR; IBRAHIM; OMAR, 2017).

A frase atribuída a William Edwards Deming, "não se gerencia o que não se mede, não se mede o que não se define, não se define o que não se entende, não há sucesso no que não se gerencia", exemplifica a relação entre os indicadores e a gestão do setor de manutenção, pois os indicadores têm um impacto significativo no departamento de engenharia clínica. O papel fundamental do indicador é definir o que deve ser medido e assim se pode começar a fazer o gerenciamento das práticas do setor. Dessa forma, é certo dizer que, para se gerenciar o setor de manutenção ou melhor, o setor de engenharia biomédica ou engenharia clínica, responsável pela manutenção, faz-se necessário um conhecimento prévio dos indicadores, a definição de quais são os mais adequados ao hospital e aplicá-los nas práticas diárias (SANTOS; LIMA; NASCIMENTO, 2020).

Portanto, o objetivo neste estudo foi realizar uma revisão bibliográfica sobre os indicadores de modo geral, com foco no setor de engenharia clínica de hospitais, trazer os principais indicadores que são usados nessa área e os resultados que o setor desses hospitais obteve na utilização dessas medições. 


\section{MATERIAIS E MÉTODOS}

Este estudo constituiu-se de uma revisão bibliográfica sobre os indicadores de manutenção de tecnologias no setor de engenharia clínica de hospitais. Na figura 1, consta um fluxograma que demonstra como foi realizado o processo de busca e organização do material bibliográfico. As buscas foram feitas em cinco bases de dados eletrônicas (Google Acadêmico, Periódicos Capes, Sciencedirect, Scopus e Springer) e foi incluído como critério de inclusão artigos publicados entre os anos de 2011 e 2021, pois, em levantamentos iniciais, identificou-se que há poucos artigos com o detalhamento sobre os indicadores de manutenção na engenharia clínica em anos anteriores a 2011.

Da mesma forma, definiu-se como idiomas de busca o português e o inglês. Logo após a escolhas dos idiomas de busca, também foram estabelecidos os termos para a pesquisa. Os termos de buscas em português foram procurados no Google Acadêmico, já os em inglês foram pesquisados no Periódicos Capes, Sciencedirect, Scopus e Springer. Os termos em português estipulados foram "Indicadores de manutenção", "Indicadores de manutenção e engenharia clínica", "Indicadores de manutenção de equipamentos médicos hospitalares", "Indicadores chave de performance", "Indicadores chave de desempenho na manutenção". Já para o inglês foram: "Maintenance indicators", "Maintenance and clinical engineering", "Hospital medical equipament maintenance indicators", "Key performace indicators", "Key performace indicators maintenance". Após fazer a seleção dos artigos de acordo com os critérios de inclusão, para definir os indicadores de manutenção, foram revisados e feita uma seleção dos indicadores apresentados nos artigos, e uma listagem dos indicadores foi montada.

Para fazer a seleção dos indicadores, foi usada uma metodologia de divisão dos indicadores em classes. Cardoso e Calil (2000), na engenharia clínica, dividiram os indicadores em três classes: classe dos indicadores temporais, classe dos indicadores de qualidade e classe dos indicadores de custo. Dessa forma, para melhorar a análise de cada um, foi seguida essa divisão. Para selecionar os indicadores temporais, foram levados em conta alguns fatores, como o tempo entre a ocorrência do problema ou parada do equipamento, identificação do que está acontecendo e a solução dessa falha. Outro fator que vale ressaltar são as horas entre a solicitação da manutenção corretiva e a realização do serviço. Para os indicadores de custo, os resultados são analisados, mensurados e divulgados, como a taxa de depreciação do equipamento, valores das manutenções corretivas que o foram feitas no equipamento e valor que o equipamento gera por mês.

Já para os indicadores de qualidade, informações resultantes do reparo e do equipamento precisam ser analisadas: se o equipamento já foi ajustado, o tempo que o equipamento estava funcionando entre cada manutenção, total de vezes que o equipamento mostrou falhas mais críticas resultando a parada. 
Figura 1 - Fluxograma da metodologia usada para a pesquisa

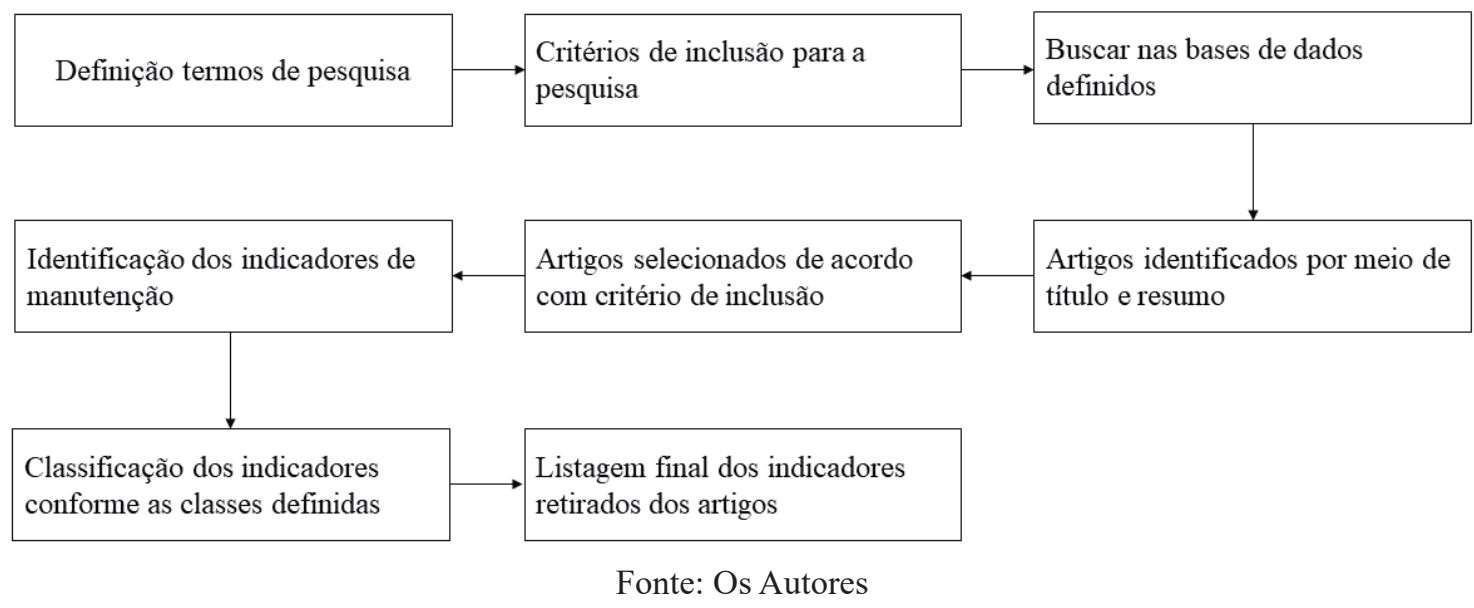

\section{RESULTADOS E DISCUÇÃO}

Após selecionar os indicadores, eles foram divididos de acordo com as classes citadas na metodologia. Foram selecionados quatorze indicadores de tempo, treze indicadores de custo e quinze indicadores de qualidade (Quadro 1).

Os indicadores de manutenção na engenharia clínica são fundamentais para melhorar o desempenho das manutenções, redução de tempo de indisponibilidade do equipamento, custos e desperdícios (SANTOS; LIMA; NASCIMENTO, 2020). Manutenções em excesso, transportes de equipamentos em vão, esperas, ineficiência na administração de estoques, pouca utilização da mão de obra e gerenciamento inadequado de informações são alguns tipos de desperdícios que são vistos na manutenção (MOSTAFA et al., 2015; MOSTAFA; DUMRAK; SOLTAN, 2015).

Os indicadores, como abordado anteriormente, dividem-se em: indicadores de tempo, indicadores de custo e indicadores de qualidade. É recomendável que pelo menos um indicador de cada classe seja utilizado dentro do processo de gestão, pois todo setor do hospital deve apresentar um serviço de alta qualidade, deve ser dinâmico e se adaptar aos prazos internos do hospital e estar em acordo com a realidade financeira do hospital e maximizar os seus recursos, sejam eles humanos, sejam materiais.

Quadro 1 - Indicadores selecionados de acordo com classe.

\begin{tabular}{|l|l|l|}
\hline \multicolumn{1}{|c|}{ INDICADORES DE TEMPO } & \multicolumn{1}{c|}{ INDICADORES DE CUSTO } & \multicolumn{1}{|c|}{ INDICADORES DE QUALIDADE } \\
\hline $\begin{array}{l}\text { Tempo de inatividade/parada do equipa- } \\
\text { mento }\end{array}$ & Custo de manutenção interna & Eficácia geral do equipamento \\
\hline Backlog de manutenção & Custo de manutenção externa & Ordem de serviço/equipamento \\
\hline Tempo médio para reparos (MTTR) & Custo da manutenção corretiva & Ordem de serviço (OS)/mês \\
\hline Tempo médio entre falhas (MTBF) & Custo da manutenção preventiva & Ordem de serviço/técnico \\
\hline Tempo de atividade & Taxa de depreciação & Taxa de disponibilidade por equipamento \\
\hline Tempo de manutenção corretiva & Renda/Custo gerado por mês & Reincidência de manutenção corretiva \\
\hline Atrasos na manutenção & Porcentagem de horas extras & Planejamento da manutenção \\
\hline Tempo de atendimento/tempo de resposta & Custo da mão de obra & Eficiência da mão de obra \\
\hline
\end{tabular}




\begin{tabular}{|c|c|c|}
\hline Horas de manutenção corretiva/OS & Custo da manutenção geral/leito & Qualidade da execução \\
\hline Horas de manutenção/leito & $\begin{array}{l}\text { Custo da manutenção geral de um equipa- } \\
\text { mento/custo de aquisição do equipamento }\end{array}$ & Disponibilidade do equipamento \\
\hline Horas trabalhadas/horas pagas & $\begin{array}{l}\text { Custo do departamento de manutenção/ } \\
\text { total de equipamento }\end{array}$ & Confiabilidade \\
\hline $\begin{array}{l}\text { Total de técnicos hora gastos em reparos de } \\
\text { emergência }\end{array}$ & $\begin{array}{l}\text { Investimentos financeiros em treinamento } \\
\text { por técnico }\end{array}$ & Manutenibilidade \\
\hline $\begin{array}{l}\text { Total de horas paradas por intervenção da } \\
\text { preventiva }\end{array}$ & $\begin{array}{l}\text { Investimento em horas de treinamento por } \\
\text { técnico }\end{array}$ & $\begin{array}{l}\text { Manutenção preventiva executada/Manu- } \\
\text { tenção preventiva planejada }\end{array}$ \\
\hline \multirow[t]{3}{*}{ Tempo médio de organização } & & OS fechadas/OS aberta \\
\hline & & Segurança do equipamento/paciente \\
\hline & & Manutenção corretiva \\
\hline
\end{tabular}

Fonte: Os Autores

\section{INDICADORES DE CUSTO}

A classe dos indicadores de custo abrange o custo de manutenção, que é o cálculo de quanto o estabelecimento gasta com manutenções; custo da aquisição do equipamento, âmbito no qual o gestor analisa fabricantes, modelos e valores para adquirir. Muitas vezes, é estabelecido um valor a ser gasto com manutenções, para fazer uma relação de quanto é gasto com manutenções e o custo para comprar um equipamento novo de modelo igual.

Uma pesquisa realizada por Indanza e colaboradores (2019) teve por objetivo verificar a viabilidade de implementação de um método baseado em evidências, ou seja, com base no histórico de falhas para manutenção de um hospital de alta complexidade com uma vasta e variada tecnologia. O processo foi divido por etapas, a primeira etapa foi classificar as ordens de serviços (OS) por meio de um conjunto de códigos e a segunda etapa constituiu por um projeto de um novo conjunto de indicadores para avaliar o desempenho da manutenção dos equipamentos.

Foram realizadas comparações entre dois indicadores, por exemplo: o custo de manutenção externa e o custo de manutenção interna de um determinado equipamento. Notou-se que, durante um determinado período, o custo da manutenção externa apresentava um média de 93,03\% do custo total. Depois de notarem essa porcentagem, a política de manutenção mudou, e os técnicos internos começaram a fornecer a manutenção. Com isso, a manutenção externa caiu para $69,48 \%$ do custo total, ajuste que, na política com base nas evidências de trabalho, mostrou uma melhora econômica.

Dos Santos e Do Valle Dallora (2019) propuseram para um hospital público universitário um conjunto de indicadores de desempenho para o setor de engenharia clínica. Eles fizeram uma pesquisa bibliográfica dos indicadores de engenharia clínica citados na literatura e, a partir dessa pesquisa, elaboram um questionário com as características que um bom indicador deve conter, que foram pertinência do indicador para a gestão, confiabilidade do indicador, viabilidade do indicador, sensibilidade do indicador e simplicidade de algoritmo de cálculo. Esse questionário foi aplicado em gestores do setor de engenharia clínica de instituições, docentes e especialistas para a avaliação. 
Após aplicar o questionário, avaliou-se a porcentagem do nível de concordância dos profissionais e, a partir disso, foram selecionados quatorze indicadores. Analisou-se o indicador custo de manutenção/custo de aquisição, que pode indicar a desativação do equipamento, mas, como é uma instituição pública, depende da verba para a aquisição e a quantidade de atendimento. Outro indicador analisado foi o tempo médio de reparos, que também depende de verbas e peças do fornecedor.

A partir de levantamentos, observou-se que, como as instituições públicas dependem de verbas e impasse burocráticos, surgem problemas difíceis de serem solucionados e que podem afetar também indicadores relacionados à produtividade dos serviços de engenharia clínica. Outro ponto observado foi que não existe uma definição de quais indicadores usar, ou seja, vai de acordo com o que cada instituição quer avaliar e o nível de complexidade de cada uma.

Domingos (2017) realizou uma análise dos equipamentos médicos do centro cirúrgico de um hospital. O estudo foi observacional do caráter quantitativo e mensurou os indicadores de tempo, qualidade e custos. Nos indicadores de custo, foi avaliado o custo de manutenção corretiva por equipamento, custo de manutenção preventiva por equipamento e custo de manutenção geral por aquisição.

O custo de manutenção corretiva de alguns equipamentos variou bastante, alguns desses valores são mostrados a seguir. A troca da caixa de comandos do equipamento custou $\mathrm{R} \$ 900,00$, o equipamento não estava ligando e os técnicos do setor não conseguiram solucionar o problema, uma empresa externa foi contratada para realizar o procedimento e custou $\mathrm{R} \$ 5.000,00$, outro procedimento custou R\$13.638,34 para a troca de um cabo SG do equipamento. No custo da manutenção preventiva, apenas um equipamento apresentou custo no valor de R $\$ 377,59$, que foi a troca de uma peça para garantir ao equipamento um bom funcionamento. No custo de manutenção geral, somente dois equipamentos tiveram uma despesa de manutenção de pouco mais de $10 \%$ do seu valor de aquisição.

\section{INDICADORES DE TEMPO}

A classe dos indicadores de tempo aborda o tempo de parada do equipamento. Esse indicador apresenta o tempo calculado em horas que o equipamento parou de funcionar devido a alguma falha e o tempo médio entre falhas, que mede o índice de falhas não previstas, que podem ser do software ou algum defeito da fabricação do equipamento. Esse indicador pode ser medido em horas, dias, semanas ou meses.

Os indicadores de manutenção estão sendo aplicados para conhecimento do efeito da idade do parque tecnológico e como isso afeta os processos e tempos de manutenção. Em um trabalho realizado por Oshiyama et al. (2014), os autores descrevem um estudo realizado na Universidade de Campinas, em que 2134 equipamentos foram estudados, sendo estes divididos entre três classes de idade. Os indicadores usados foram: número de OS de manutenção corretiva (MC); horas de manutenção corretiva (HM) e custo total para OS de manutenção corretiva (CM). Conforme apresentado na figura 2, pode-se observar, de forma clara e objetiva, que um parque tecnológico mais velho acarreta maior 
despendimento financeiro, necessita de maior número de horas de manutenção e impacta em mais tempo de máquina parada (maior número de OS de $\mathrm{MC}$ ). Esses resultados são importantes para o setor de manutenção demonstrar a necessidade de atualização do parque, bem como a demanda de mão de obra e peças para o parque, justificando os gastos totais praticados.

Figura 2 - Média dos valores para cada indicador em relação a idade dos equipamentos. Número de OS de manutenção corretiva (CM); horas de manutenção corretiva (HM) e custo total para OS de manutenção corretiva (CM)
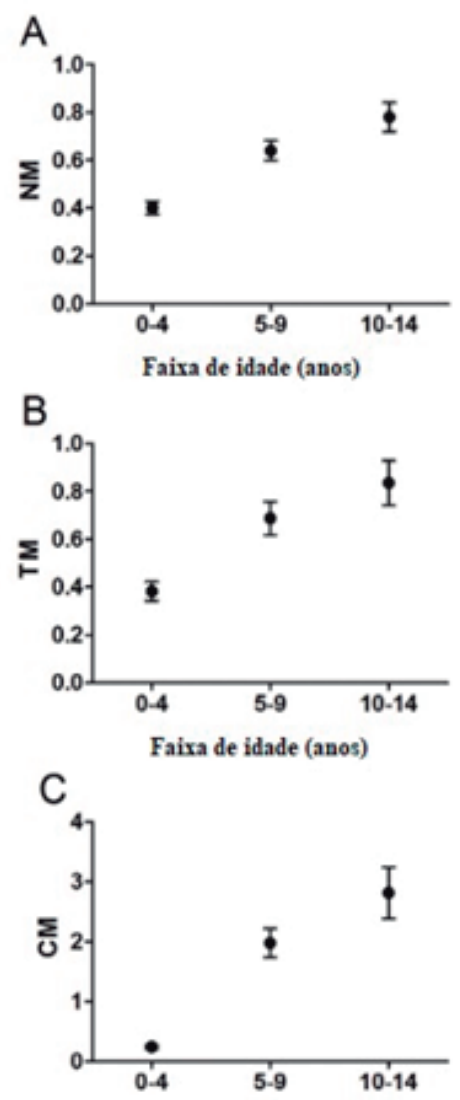

Fonte: adaptado de Oshiyama et al. (2014).

Os indicadores de tempo analisados por Domingos (2017) foram o tempo médio de manutenção corretiva (TMMC) e tempo médio entre falhas (MTBF). O TMMC, na maioria dos casos, foi baixo, mostrando que o setor de engenharia clínica tem eficiência na solução dos problemas e na realização rápida das manutenções corretivas. Os casos que eles não conseguiam cumprir eram devido a alguns problemas descritos nas ordens de serviço.

No MTBF, quanto maior ele for, melhor o estado do equipamento médico, pois é o tempo de demora entre uma manutenção corretiva e outra a ser realizada. Esse indicador apresentou resultados diferentes entre os equipamentos, alguns equipamentos tiveram o MTBF baixo, ou seja, eles precisaram de manutenção em um tempo menor, e os outros equipamentos tiveram um valor que já era esperado, mas com algumas exceções. 
Moura (2019) apresenta uma análise dos indicadores de manutenção corretiva dos desfibriladores/cardioversores de um hospital. Para isso, foram usados os indicadores de tempo médio de reparo (MTTR), MTBF e disponibilidade. Na análise do MTBF, percebeu que a diferença não foi muito relevante, pois, no cálculo, não foram excluídos os dias que o equipamento ficou indisponível. Por outro lado, no MTTR, observou-se valores elevados, possivelmente, devido à demora na compra das peças para o conserto do equipamento.

\section{INDICADORES DE QUALIDADE}

A classe dos indicadores de qualidade engloba as manutenções preventivas que foram realizadas e as que iram ser realizadas. São observadas e calculadas em um período em que o responsável possa verificar se o plano de manutenções está sendo executado como o esperado.

Em pesquisa realizada por De Souza e colaboradores (2012), em um hospital público do Brasil, foi possível observar grandes mudanças com a aplicação de processos para o monitoramento e gestão dos processos de manutenção. Como pode ser visto na figura 3, ao longo do período de implementação do setor de manutenção, os processos deixaram de ser exclusivamente corretivos para se tornarem parcialmente preventivos e corretivos. Esse acompanhamento do indicador de $\mathrm{MC} / \mathrm{MP}$ permite às equipes avaliarem a presença ativa do setor em práticas sistemáticas de controle do equipamento e da qualidade de implementação dos serviços. A presença da MP em detrimento a MC é vantajosa ao hospital, pois tende a reduzir o tempo de equipamento parado, maximizando as práticas em saúde.

No mesmo artigo, os autores comparam os indicadores de porcentagem de manutenção externa e porcentagem de manutenção interna e seu impacto financeiro ao longo do período avaliado. Segundo os autores, a estimativa de economia acumulada nos 10 anos de avaliação é de R\$7.688.056,57 (sete milhões, seiscentos e oitenta e oito mil e cinquenta e seis reais com cinquenta e sete centavos). Esse dado demonstra claramente como um indicador pode mostrar de forma clara e objetiva a importância de um setor de manutenção estruturado e com conhecimento do que se é realizado por ele. Na figura 3, constam os percentuais das manutenções.

Em relação a indicadores de qualidade, também pode-se citar o trabalho apresentado por Sezdi (2016), em que o autor descreve as OS por equipamentos e como elas sofreram alteração após o setor desenvolver metodologias de manutenção preventiva. Como pode ser observado na figura 4, os desfibriladores deixaram de apresentar OS para problemas relacionados a bateria, BPM, eletrodos e pás. Também é interessante observar que as OS por não funcionamento dos equipamentos não ocorreram mais depois da implementação do serviço de manutenções preventivas. 
Figura 3 - Evolução dos percentuais de manutenção interna, externa, corretivas e preventivas no HC-UFU, no período de 2001 a 2010.

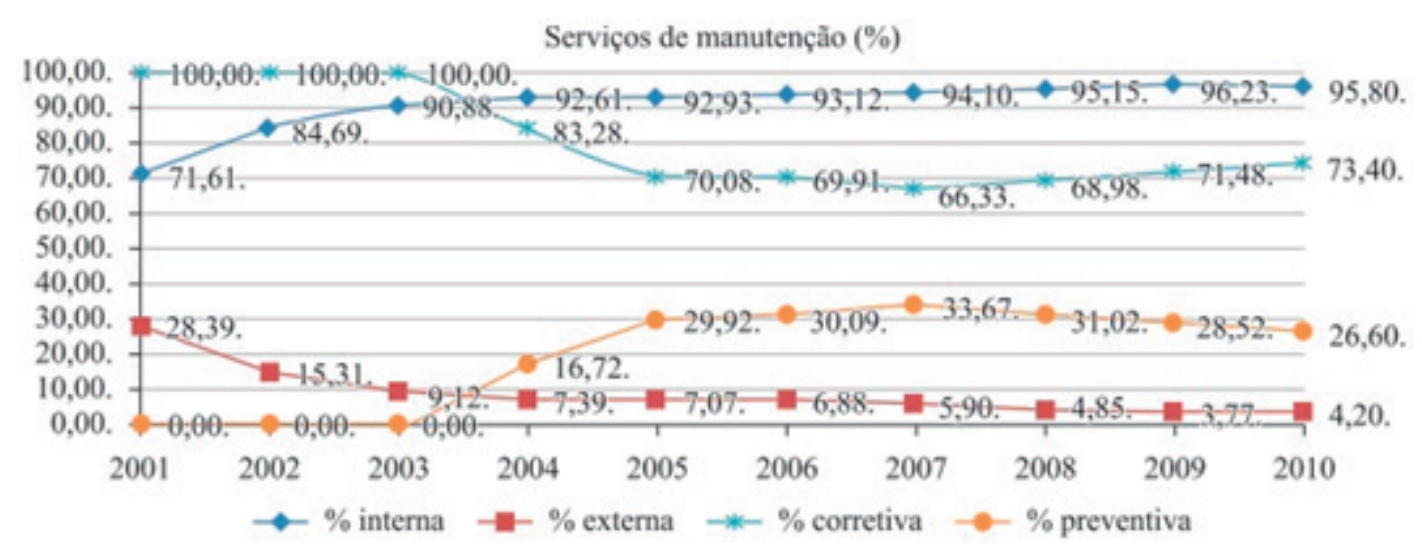

Fonte: De Souza et al. (2012).

Figura 4 - Falha de desfibriladores antes e após a implementação da manutenção preventiva.

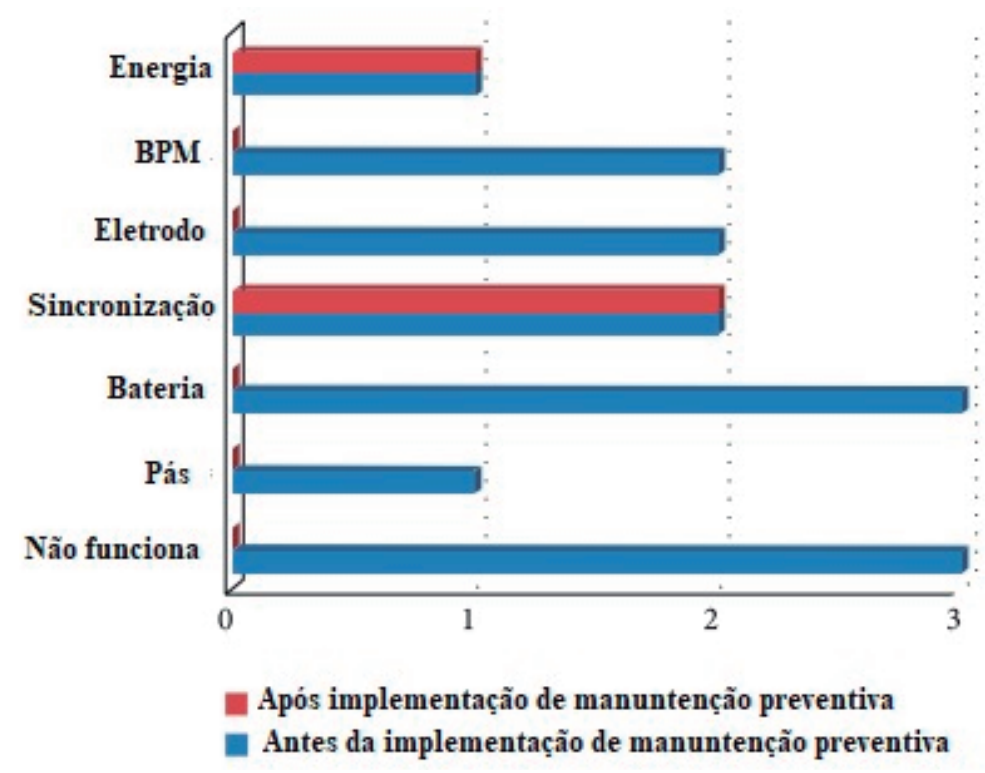

Fonte: adaptado de Sezdi (2016).

Nos indicadores de qualidade, Domingos (2017) analisou a manutenção preventiva realizada (MPR) x manutenção preventiva desejada (MPD), número de ordens de serviços fechadas x número de ordens de serviço abertas e número de ordens de serviço total em um equipamento por mês. No indicador MPR x MPD, apenas em dois equipamentos foram realizadas de forma correta a quantidade de MPR, alguns equipamentos tiveram mais manutenções preventivas que outros, por exemplo, aconteceu de um equipamento não ter nenhuma manutenção preventiva realizada e outros terem manutenções realizadas a mais.

Em relação ao número de ordens de serviços fechadas $\mathrm{x}$ abertas, percebeu que, em dois anos, elas foram fechadas e concluídas assim que os problemas eram solucionados, mas vale ressaltar que o período analisado foi definido e não se teve acesso aos dados do período médio que as OSs ficaram 
abertas, para saber o tempo em que o setor consegue solucionar o problema. No que se refere ao número de OS total de um equipamento por mês, foram consideradas as manutenções corretivas e preventivas realizadas em dois anos, o sistema não permite calcular o período que o equipamento ficou parado e, dependendo do problema que acontece, o equipamento pode estar em uso com restrições. Portanto, todos os equipamentos tiveram manutenções corretivas e preventivas, mas teve equipamentos em que foram realizadas mais manutenções preventivas do que outros, em que foram realizadas mais manutenções corretivas.

No indicador de disponibilidade, Moura (2019) analisou os equipamentos que tiveram mais disponibilidade, com exceção dos que apresentaram 100\%, e obteve resultados de equipamentos que tiveram 99,98\% de disponibilidade. Um deles ficou o mínimo de tempo na manutenção e foi feita apenas uma revisão geral quando ele foi para manutenção, um segundo equipamento teve a porcentagem de 73,51\% de disponibilidade e ficou 765 dias na manutenção, mas isso se deu por causa da demora na compra de novas pás para consertar o dispositivo.

\section{CONCLUSÃO}

Pode-se constatar que os indicadores são medições que colaboram muito para o desempenho do setor. Percebe-se também que existem muitos indicadores de gestão, que variam muito, pois são elaborados de acordo com a necessidade e o objetivo do setor. Não existe um padrão de quais serão utilizados, eles precisam ser adaptáveis à realidade do estabelecimento e sempre fazer uma análise crítica dos seus resultados ao longo do tempo. A rastreabilidade nos indicadores na área de engenharia clínica oferece segurança tanto aos profissionais de saúde como aos pacientes, além do custo-efetividade no monitoramento dos equipamentos que estão em processo de avaliação.

Conforme foi discutido nas aplicações, os indicadores, quando usados com eficiência, mostram bons resultados, pois, antes de serem implementados, o estabelecimento realiza um estudo crítico de quais objetivos o setor quer atingir e, com isso, elabora os seus indicadores de acordo com o objetivo, focando na melhoria do setor, para que os profissionais consigam extrair as informações baseadas nos objetivos. À medida em que os indicadores de custo, tempo e qualidade se cruzam na análise minuciosa e prática dos equipamentos, estas sinalizam para os setores, inclusive administrativo, as diferentes possibilidades de intervenção na vida útil do equipamento, o que permite que sejam definidos processos capazes de aliar qualidade do parque tecnológico, gestão financeira sustentável e segurança dos pacientes e profissionais.

Nas aplicações citadas, observou-se que os indicadores relacionados às características de produtividade e agilidade na resolução dos problemas tiveram um bom desempenho de concordância. Outro ponto importante que pode ser citado é manter a equipe sempre atualizada e motivada para que, juntos, possam fazer uma boa gestão das medições no setor, fazendo com que esses profissionais 
enxerguem a importância de sua participação nos feedbacks apresentados, na busca das metas e no trabalho de análise dos resultados.

\section{REFERÊNCIAS}

AGÊNCIA NACIONAL DE VIGILÂNCIA SANITÁRIA. Resolução da diretoria colegiada - RDC no 509, de 27 de maio de 2021. Disponível em: https://bit.ly/3nk8Md3. Acesso em: 18 jun. 2021.

ARAÚJO, C. C. Análise e simulação de processos de manutenção de equipamentos médicos no serviço de electromedicina. Porto: Instituto Superior de Engenharia do Porto, 2020.

CARDOSO, G. B.; CALIL, S. J. Estudo do processo de análise de referência aplicado à engenharia clínica e metodologia de validação de indicadores de referência. In: XVII CONGRESSO BRASILEIRO DE ENGENHARIA BIOMÉDICA, 2000, Florianópolis. Anais [...]. Florianópolis: SBEB, 2000. p. 482-487.

DE SOUZA, D. B. et al. Avaliação econômica da implantação de um serviço de Engenharia Clínica em hospital público brasileiro. Revista Brasileira de Engenharia Biomédica, v. 28, n. 4, p. 327-336, nov., 2012. Disponível em: https://bit.ly/3vBZOeW. Acesso em: 30 abr. 2021.

DOMINGOS, D. C. L. Análise dos indicadores referentes aos equipamentos médico-hospitalares do centro cirúrgico de um hospital privado da cidade de Natal. 2017. 50 f. Trabalho de Conclusão de Curso (Centro de Tecnologia Departamento de Engenharia Biomédica) - Universidade Federal do Rio Grande do Norte, Natal, 2017.

DOS SANTOS, R. M.; DO VALLE DALLORA, M. E. L. Avaliação de indicadores de desempenho da área de engenharia clínica. Medicina, Ribeirão Preto, v. 52, n. 1, p. 34-46, 2019.

GONNELLI, V.; SATTA, F.; FROSINI, F.; IADANZA, E. Evidence-based approach to medical equipment maintenance monitoring. IFMBE Proceedings. [S. l.]: Springer Verlag, 2017. v. 65, p. 258-261. Disponível em: https://doi.org/10.1007/978-981-10-5122-7_65. Acesso em: 15 mar. 2021.

IADANZA, E.; GONNELLI, V.; SATTA, F.; GHERARDELLI, M. Evidence-based medical equipment management: a convenient implementation. Medical and Biological Engineering and Computing, v. 57, n. 10, p. 2215-2230, 2019. Disponível em: https://doi.org/10.1007/s11517-019-02021-x. Acesso em: 20 jan. 2021. 
MAGGI, N.; ADORNETTO, A.; SCILLIERI, S.; URBINA, E. N. B.; RUGGIERO, C.; GIACOMINI, M. Medical equipment replacement prioritisation: A comparison between linear and fuzzy system models. Studies in Health Technology and Informatics. IOS Press, p. 1538-1539, 2019. Disponível em: https://doi.org/10.3233/SHTI190523. Acesso em: 27 jun. 2021.

MOSTAFA, S.; DUMRAK, J.; SOLTAN, H. Lean Maintenance Roadmap. Procedia Manufacturing, v. 2, p. 434-444, 2015. Disponível em: https://doi.org/10.1016/j.promfg.2015.07.076. Acesso em: 25 abr. 2021.

MOSTAFA, S.; LEE, S. H.; DUMRAK, J.; CHILESHE, N.; SOLTAN, H. Lean thinking for a maintenance process. Production and Manufacturing Research, v. 3, n. 1, jan., 2015, p. 236-272. Disponível em: https://doi.org/10.1080/21693277.2015.1074124. Acesso em: 19 jun. 2021.

MOURA, Y. R. Análise de indicadores de manutenção dos desfibriladores/cardioversor do Hospital de Clínicas de Uberlândia da Universidade Federal de Uberlândia. 2019.

NOVAES, H. M. D.; DE SOÁREZ, P. C. Health Technologies Assessment: origins, development, and current challenges. In the international and Brazilian scenarios. Cadernos de Saúde Pública, v. 36, n. 8, 2020. Disponível em: https://doi.org/10.1590/0102-311X00006820. Acesso em: 20 jun. 2021.

OMAR, M. F.; IBRAHIM, F. A.; OMAR, W. M. S. W. Key performance indicators for maintenance management effectiveness of public hospital building. MATEC Web of Conferences. EDP Sciences, 2017. p. 01056.

ORTIZ, A.; ARAUJO, E. Sistema Inteligente de Parque Tecnológico Médico-Hospitalar para Substituição de Equipamentos Médicos. In: CONGRESSO BRASILEIRO DE ENGENHARIA BIOMÉDICA, 2020, Vitória. Anais [...]. Vitória: SBEB, 2020. Disponível em: https://bit.ly/3G331ZL. Acesso em: 19 jun. 2021.

OSHIYAMA, N. F. et al. Medical equipment classification according to corrective maintenance data: A strategy based on the equipment age. Revista Brasileira de Engenharia Biomedica, v. 30, n. 1, p. 64-69, 2014.

SANTOS, L.; LIMA, A.; NASCIMENTO, G. Priorização de Desperdicios na Manutenção de Equipamentos Médicos em Operações Hospitalares. RAHIS- Revista de Administração Hospitalar e Inovação em Saúde, Belo Horizonte, v. 17, n. 4, p. 1983-5205, 2020. Disponível em: https://doi.org/ 10.21450/rahis.v17i4.6455. Acesso em: 19 jun. 2021. 
SHAMAYLEH, A.; AWAD, M.; FARHAT, J. IoT Based Predictive Maintenance Management of Medical Equipment. Journal of Medical Systems, v. 44, n. 4, p. 1-12, 2020. Disponível em: https:// doi.org/10.1007/s10916-020-1534-8. Acesso em: 20 abr. 2021.

SEZDI, M. Two Different Maintenance Strategies in the Hospital Environment: Preventive Maintenance for Older Technology Devices and Predictive Maintenance for Newer High-Tech Devices. Journal of Healthcare Engineering, v. 2016, 2016. Disponível em: https://doi.org/10.1155/2016/7267983. Acesso em: 25 abr. 2021.

SILVA, D. C. S.; LUCENA, A. D.; TOSCANO, J. Estudo de indicadores chave de desempenho em manutenção e construção de um dashboard em uma indústria do ramo petrolífero. In: XXXV ENCONTRO NACIONAL DE ENGENHARIA DE PRODUCAO: PERSPECTIVAS GLOBAIS PARAA ENGENHARIA DE PRODUÇÃO, 2015, Fortaleza. Anais [...]. Fortaleza: UFC, 2015. p. 1-20.

WANG, B. et al. Evidence-based maintenance: part I: measuring maintenance effectiveness with failure codes. Journal of Clinical Engineering, v. 35, n. 3, p. 132-144, 2010. 